\title{
Does age affect the relationship between pain and disability? A descriptive study in individuals suffering from chronic low back pain
}

\begin{abstract}
Background: Previous studies have revealed a weak to moderate relationship between pain and disability in individuals suffering from low back pain (LBP). However, to our knowledge, no studies have evaluated if this relationship is different between young and older adults.
\end{abstract}

Purpose: The objective of this descriptive, cross-sectional study, was to determine if the relationship between LBP intensity and physical disability is different between young and older adults.

Methods: Pain intensity (measured with a visual analog scale) and physical disability scores (measured with the Oswestry Disability Index) were collected from the medical files of 164 patients with LBP. Separate Pearson correlation coefficients were calculated between these 2 variables for young (mean age $40 \pm 6$ years, $n=82$ ) and older $(62 \pm 9$ years, $n=82$ ) individuals and a Fisher $r$-to-z transformation was used to test for group differences in the strength of the relationship. Linear regression analyses were also performed to determine if the slope of the association was different between the 2 groups.

Results: There was a significant and positive association between pain intensity and disability for both young and older individuals. However, the correlation was stronger in 
the young group $(\mathrm{r}=0.66 ; \mathrm{p}<0.01)$ compared to the older group $(\mathrm{r}=0.44 ; \mathrm{p}<0.01)$ (Fisher $Z=2,03 ; p<0.05)$. The linear regression model also revealed that the slope of the relationship was steeper in the young group $(\mathrm{p}<.05)$.

Conclusion: Although both young and older individuals showed a significant association between pain intensity and disability, the relationship between these 2 variables was more tenuous in older individuals than in young patients. Future research is essential to identify the factors underlying this age-related difference.

Keywords: pain, chronic pain, low back pain, disability, aging 


\section{INTRODUCTION}

Low back pain (LBP) is one of the leading causes of absenteeism from work, worldwide. ${ }^{1}$ According to the Global Burden of Disease (GBD) Project of the World Health Organization, LBP is now the most disabling pathology, well ahead of 290 other health conditions. ${ }^{2,3}$ Not surprisingly, this condition is also very costly for modern societies. For example, in the United States, the total expenses associated with LBP are estimated to be between $\$ 100$ and $\$ 200$ billion annually, with the majority of the costs attributable to decreased wages and productivity. ${ }^{4}$

More than $80 \%$ of the population will be affected by LBP during their lifetime and, within a one-year period basis, $19 \%$ will experience a new or recurrent episode of LBP. 1,5 Interestingly, data obtained from the GBD initiative reveals that the chances of suffering from LBP are higher in middle-aged individuals, with peak prevalence rates reported in individuals aged between 60 and 65 years old. ${ }^{5}$ Although the prevalence of LBP slightly decreases after 65 years of age, 5 individuals older than 65 tend to be more frequently affected by severe LBP than their younger counterparts. ${ }^{6}$

According to Salvetti and colleagues, the prevalence of disability - defined by the authors using an Oswestry disability index score $\geq 20$ and absence from work for at least 30 days in the past year - is estimated at $65 \%$ in patients suffering from chronic low back pain (CLBP). ${ }^{7}$ In the past years, the relationship between LBP and physical function has been studied by many researchers, with results showing the presence of a weak to moderate relationship between LBP intensity and disability. ${ }^{8-10}$ However, these studies are generally conducted on middle-aged individuals or on age-heterogeneous samples, and it is still 
unknown if the weak to moderate relationship observed between pain intensity and disability is the same in young and older individuals.

Recent data stemming from epidemiological studies suggest that LBP could affect the physical function of young and older individuals differently.,3 For instance, based on the data obtained from the GBD initiative, Buchbinder and colleagues reported that the disability burden per capita - calculated by multiplying the number of cases per 100,000 individuals by the average duration of the condition and by the disability weight - is higher in older age groups. ${ }^{2}$ In their study, Buchbinder and colleagues investigated the effect of age on the disability burden of individuals with LBP; however, the relationship between LBP intensity and physical disability was not directly explored. It is therefore impossible to know if the increased disability burden reported by Buchbinder and colleagues in older individuals can be attributable to differences in the relationship between pain and disability in young and older individuals, or simply to the fact that the latter experience more pain than the former. 6,11

Information regarding the effect of age on the relationship between LBP and disability is fundamental for rehabilitation specialists and other clinicians working with individuals affected by CLBP. Healthcare providers and policy makers generally assume that the weak to moderate relationship between pain and disability described in the general population is the same in young and older individuals. To our knowledge, no previous study has assessed whether the relationship between LBP and disability is affected by age.

The objective of the present study was to fill this knowledge gap by determining if the strength and the slope of the relationship between LBP intensity and physical disability 
is different in young and older individuals.

\section{METHODS}

\section{Data collection and participants}

In order to achieve our objectives, a cross-sectional study design was adopted. The information, collected from the patients' medical files, included the intensity of the LBP at the moment of consultation [measured with a visual analog scale (VAS) of $10 \mathrm{~cm}(0=$ no pain, $10=$ worst pain imaginable)] as well as the disability associated with LBP [measured with the Oswestry Disability Index (ODI) ${ }^{12}$ ]. In order to eliminate the potential confounding effect that surgery could have on the results, we only considered VAS and ODI scores obtained before surgery. Demographic (age, sex) and clinical information (diagnosis, treatments received) were also collected.

Clinical data were collected from patients with LBP seen at the Spine Clinic of the Centre hospitalier universitaire de Sherbrooke (CHUS), a secondary and tertiary level of care clinic, between August 2004 and April 2010. Patients were all seen by the same orthopedic surgeon (FC). To be included in the study, patients had to be aged 18 years or older and have suffered from musculoskeletal LBP for over 6 months. Of the 547 patient files first identified, 292 were incomplete and were therefore exclud ed from the analyses. Among the 255 remaining files, 27 were excluded because of spinal pain of cervical origin, 28 because of pain from a neoplasic origin, and 27 because of a past back surgery. Nine other patient files were excluded because they were experiencing no pain at the moment of consultation. Thus, 164 patients met the eligibility criteria and were included in the analyses (see Figure 
1). The study's procedures were approved by the Research Ethics Board for Human Subjects of the CHUS and the rights of the participants were protected at all time.

\section{Statistical analysis}

To evaluate the effect of age on the relationship between LBP and disability, correlation between these variables was compared between young and older individuals. The 2 age groups were created by splitting the sample into 2 equal groups using a median split approach. This resulted in the creation of 2 equal groups: a young group $(n=82)$, aged between 22 and 48 years old, and an older group $(n=82)$, aged between 49 and 90 years old. Separate Pearson correlation coefficients for young and older individuals were calculated to estimate the strength of the relationship in the 2 age groups, and a Fisher $r$-to$\mathrm{z}$ transformation was used to test for differences between the 2 correlation coefficients. ${ }^{13,14}$ This allowed us to determine if the strength of the association between LBP intensity and disability was different in young and older individuals.

A linear regression analysis was also performed, with the variables "Pain", "Age group" and interaction between pain and age group ("Pain X Age group") entered as independent variables and the variable "Disability" entered as the dependent variable. This allowed us to determine if the slope of the association was different between young and older individuals. All statistical analyses were performed using SPSS 17.0 for Windows (SPSS Inc., Chicago, IL). The significance level was set at $\mathrm{p}<0.05$.

\section{Results}


Patients' characteristics

Patients' characteristics are presented in Table 1. As can be seen in this table, there was no difference between the 2 age groups for pain intensity and physical disability. The proportion of men tended to be higher in the young group than in the older group. However, the difference did not reach statistical significance $(\mathrm{p}=.06)$. The main diagnoses were herniated discs and multi-scale degenerative disc disease in the young group and central stenosis and multi-scale degenerative disc disease in the older group. The mean pain intensity and disability scores were high in both groups (all VAS scores > 6; all ODI scores $>$ 22).

\section{Correlation analyses}

There was a significant and positive association between pain intensity and physical disability in the entire group $(\mathrm{n}=164, \mathrm{r}=0.55 ; \mathrm{p}<0.01)$. The correlation analyses, performed after dividing the sample into 2 equal groups, showed that the association was still significant in both the young and older group. However, the strength of the correlation was stronger in the young group $(r=0.66 ; \mathrm{p}<0.01)$ compared to the older group $(\mathrm{r}=0.44$; $\mathrm{p}<0.01$ ) (see Figures 2A and 2B). The difference between the young and older group was confirmed by Fisher r-to-z analysis $(\mathrm{Z}=2.03, \mathrm{p}<0.05)$.

\section{Linear regression model}

The linear regression model revealed the presence of a significant "Pain X Age group" interaction $(\mathrm{p}<.05)$, suggesting that the slope of the pain-disability association was different in the 2 groups. Analysis of the regression lines revealed that the relationship 
between pain and disability was steeper in the young group compared to the older group (see Figure 3). To further examine the effect of age on the relationship between pain and disability, a second regression model was performed with the variable "Age" left undichotomized. The results showed that, contrary to the previous regression model, there was no significant interaction between the "Pain" variable and the "Age" variable $(p=0.46)$.

\section{DISCUSSION}

The aim of the present study was to determine if the relationship between pain intensity and disability is different between young and older individuals suffering from LBP. We found that the relationship between these2 variables was significantly higher in young individuals compared to older individuals.

The difference between the young and older group was revealed by the Fis her r-to-z test, as well as by significant "Pain X Age group" interaction of the linear regression model. On one side, the Fisher r-to-z test confirmed that the strength of the relationship between pain intensity and disability was different in the 2 age groups. More specifically, the paindisability relationship was stronger in the young group compared to the older group, suggesting that pain explains a greater portion of the variance of disability in young individuals. Although the exact reason for this group difference remains unknown, many hypotheses can be proposed. For example, it is possible that factors other than pain (e.g., pain self-efficacy, mood, muscle strength, fitness level) could contribute to a greater extent to the perceived level of disability in older individuals than it does in young individuals, ${ }^{15-17}$ hence reducing the proportion of variance explained by pain intensity in this portion of the 
population.

On the other hand, the significant "Pain X Age group" indicates that the slope of the relationship is different between young and older individuals. It is important to note that the slope of the relationship was steeper in the young group, suggesting that an increase in pain produces more disability in young than in older individuals. According to the linear regression equations, the highest group differences are observed for individuals with high and low pain scores. For example, in patients with a VAS pain score of 1.0, the group difference in ODI score is estimated at 5.2, compared to 1.3 in individuals with a VAS score of 5.0 and 3.5 in individuals with a VAS score of 10.0. Although no single agreed-upon minimal clinically important difference (MCID) score was established for the ODI (the proposed MCID scores range from 5 to 17 points ${ }^{18-23}$ ), the relatively small group differences observed suggest that the significant "Pain X Age group" interaction noted in this study is probably of little importance, at least from a clinical point of view.

Remarkably, no interaction was found when age was used as a continuous variable. These results suggest that age does not affect the slope of the relationship in a continuous manner. Instead, it seems as if the relationship between pain and disability stays the same until a certain age is reached. Only past this point (i.e., 49 years old) would the slope of the relationship differ significantly. Worded differently, this means that the relationship between pain and disability would be similar for an individual aged 35 and an individual aged 45, but would be different for an individual aged 45 and an individual aged 55 . Interestingly, the 49 year cut-off point used in this study corresponds to the age above which the changes in muscle mass and muscle strength start to become more 
pronounced. ${ }^{24-26}$ These observations support the idea that factors other than pain (e.g., muscle strength) would play an increasingly important role in the physical functioning of older individuals.

Relationship between pain and disability

Past studies have revealed the presence of a weak to moderate relationship between LBP intensity and physical disability. ${ }^{8-10}$ For instance, Bean and collaborators ${ }^{8}$ reported a Pearson correlation coefficient of 0.40 between pain intensity (measured using the pain numerical rating scale) and disability (measured using the Pain Disability Index) in a sample of 88 patients affected by CLBP. Slightly higher correlation coefficients were obtained by Chung et al. ${ }^{10}$ in their study performed on a sample of 55 patients suffering from CLBP. More specifically, Chung et al. observed a Pearson correlation coefficient of 0.49 when disability was measured with the ODI and 0.53 when disability was measured with the Roland Morris Disability Questionnaire. Similar results were obtained by Woby et al., ${ }^{9}$ who noted a Pearson correlation coefficient of 0.54 between pain intensity (measured with the VAS) and disability (measured with the Roland Morris Disability Questionnaire) in 183 patients with nonspecific CLBP. These findings are coherent with the results of this study, in which we observed a Pearson correlation coefficient of 0.55 in the total patient sample.

\section{Relationship between pain and disability in young and older individuals}

To our knowledge, only one study has evaluated if the relationship between pain and disability is affected by age. ${ }^{27}$ In this study, performed by Lewandowski and colleagues in a population of children suffering from recurrent headaches, the authors noted that the 
relationship between pain intensity and physical disability (measured using the Functional Disability Index) ${ }^{28}$ was substantially higher in children aged between 8 and 12 years old ( $\mathrm{r}$ $=0.37, \mathrm{p}<0.05)$ compared to adolescents aged 13 to 16 years old $(\mathrm{r}=0.29, \mathrm{p}>0.05)$. Our results confirm and extend the results of Lewandowski et al. by showing that the effect of age on the pain-disability relationship is not limited to individuals with recurrent headaches, but is also present in individuals suffering from LBP. Future studies are necessary to determine if similar results can be found in other pain populations.

\section{Limitations}

From a statistical point of view, the decision to break a continuous variable into 2 or more categories is always questionable, notably because of the necessity of choosing one (or several) cutoff point(s) to divide the variable. In the present study, the "Age" variable was dichotomized using a median-split approach, resulting in the creation of 2 equal groups of 82 patients with LBP: one group of young individuals aged between 22 and 48 years old and one group of older individuals aged between 49 and 90 years old. This entails that the cutoff point used was driven by the characteristics of the patient sample (i.e., the age of the patients), and one can wonder if the pattern of results described in this study would have been different with a different patient sample and cutoff point. These questions are particularly pertinent when we consider the absence of significant interaction when the "Age" variable was left undichotomized and the unequal age span between the 2 groups $(26$ years for young group and 41 years old for the older group). The decision to dichotomize the "Age" variable was motivated by the objectives of the study and by the statistical analyses approach used. In particular, the Fisher r-to-z analysis (which allowed us to test if 
age affects the strength of the relationship between pain and disability) requires the creation of 2 separate groups. Given the absence of data suggesting that a certain cutoff point should be used for age dichotomization, dividing the "Age" variable by using a median-split approach appeared to be the better option.

Another important limit concerns the possible generalizability of our results. Because participants were all recruited from the Spine Clinic of the CHUS, one can wonder if the results obtained from this group of patients would also have been found in a more general LBP population. Although we cannot completely rule out the possibility that our results are limited to a certain portion of individuals suffering from LBP, this appears unlikely, given the nature of the patient population seen at the Spine Clinic of the CHUS (i.e., patients who are highly disabled who are referred for surgery, but also lighter cases of patients who are referred for a second opinion on non-surgical CLBP management). Thus, our results are probably generalizable to a large proportion of the CLBP population.

Moreover, it should be noted that several factors known to affect pain and disability were not included in the analyses. For example, it would be interesting to know if the conclusions are the same after adjusting for smoking status, ${ }^{29}$ opioid consumption, ${ }^{30}$ and psychological factors (e.g., anxiety ${ }^{31}$, catastrophizing 32 , beliefs and fears regarding current and future work tolerance ${ }^{33,34}$ ). More information regarding the characteristics of the patients (e.g., work status, access to a disability pension plan) would also have been useful in the interpretation of the current results. Unfortunately, this information was not available to the researchers.

Finally, the group differences observed in the diagnoses of the 
participants is also an important limitation. Young individuals suffered, mostly, of degenerative disc disease (DDD) and herniated discs, whereas older individuals were generally diagnosed with DDD and central stenosis. It is therefore difficult to conclude if the pattern of results observed in this study is due to the group difference in age or in diagnoses. Future studies should be wary of this potential confound.

\section{CONCLUSION}

To our knowledge, this study is the first of its kind to determine if the relationship between LBP intensity and physical disability is influenced by age. The results show that the strength and the slope of the relationship between pain and disability are different in young and older individuals. Future, ideally multicenter studies should be performed to see if these findings can be replicated and observed in other pain populations, and to determine if the differences between young and older individuals truly tend to appear after 49 years of age. 


\section{ACKNOWLEDGEMENTS}

The authors wish to thank Lise Trottier and Nathalie Carrier for their thoughtful comments on the manuscript and Janie Plourde-Gauthier for her help with data collection. 


\section{REFERENCES}

1. Cassidy JD, Cote P, Carroll LJ, Kristman V. Incidence and course of low back pain episodes in the general population. Spine (Phila Pa 1976). 2005;30(24):2817-2823.

2. Buchbinder R, Blyth FM, March LM, Brooks P, Woolf AD, Hoy DG. Placing the global burden of low back pain in context. Best Pract Res Clin Rheumatol. 2013;27(5):575-589.

3. Hoy D, March L, Brooks P, et al. The global burden of low back pain: Estimates from the global burden of disease 2010 study. Ann Rheum Dis. 2014.

4. Katz JN. Lumbar disc disorders and low-back pain: Socioeconomic factors and consequences. J Bone Joint Surg Am. 2006;88 Suppl 2:21-24.

5. Hoy D, March L, Brooks P, etal. Measuring the global burden of low back pain. Best Pract Res Clin Rheumatol. 2010;24(2):155-165.

6. Dionne CE, Dunn KM, Croft PR. Does back pain prevalence really decrease with increasing age? A systematic review. Age Ageing. 2006;35(3):229-234.

7. Salvetti Mde G, Pimenta CA, Braga PE, Correa CF. Disability related to chronic low back pain: Prevalence and associated factors. Rev Esc Enferm USP. 2012;46 Spec No:16-23.

8. Bean DJ, Johnson MH, Kydd RR. Relationships between psychological factors, pain and disability in complex regional pain syndrome and low back pain. Clin J Pain. 2013.

9. Woby SR, Roach NK, Urmston M, Watson PJ. The relation between cognitive factors and levels of pain and disability in chronic low back pain patients presenting for physiotherapy. Eur J Pain. 2007;11(8):869-877. 
10. Chung EJ, Hur YG, Lee BH. A study of the relationship among fear-avoidance beliefs, pain and disability index in patients with low back pain. J Exerc Rehabil. 2013;9(6):532-535.

11. Choiniere M, Dion D, Peng P, et al. The canadian STOP-PAIN project - part 1: Who are the patients on the waitlists of multidisciplinary pain treatment facilities? Can J Anaesth. 2010;57(6):539-548.

12. Fairbank JC, Couper J, Davies JB, O'Brien JP. The oswestry low back pain disability questionnaire. Physiotherapy. 1980;66(8):271-273.

13. Fisher RA. On the "probable error" of a coefficient of correlation decuded from a small sample. Metron. 1921;1:3-32.

14. Fisher RA. Frequency distribution of the values of the correlation coefficient in samples from an indefinitely large population. Biometrika. 1915;10(4):507-521.

15. Costa Lda C, Maher CG, McAuley JH, Hancock MJ, Smeets RJ. Self-efficacy is more important than fear of movement in mediating the relationship between pain and disability in chronic low back pain. Eur J Pain. 2011;15(2):213-219.

16. Singh MA. Exercise comes of age: Rationale and recommendations for a geriatric exercise prescription.J Gerontol A Biol Sci Med Sci. 2002;57(5):M262-82.

17. Kennis E, Verschueren S, Van Roie E, Thomis M, Lefevre J, Delecluse C. Longitudinal impact of aging on muscle quality in middle-aged men. Age (Dordr). 2014;36(4):9689-0149689-1. Epub 2014 Aug 8.

18. Maughan EF, Lewis JS. Outcome measures in chronic low back pain. Eur Spine J. 
2010;19(9):1484-1494.

19. Ostelo RW, de Vet HC. Clinically important outcomes in low back pain. Best Pract Res Clin Rheumatol. 2005;19(4):593-607.

20. Hagg O, Fritzell P, Nordwall A, Swedish Lumbar Spine Study Group. The clinical importance of changes in outcome scores after treatment for chronic low back pain. Eur Spine J. 2003;12(1):12-20.

21. Cleland JA, Whitman JM, Houser JL, Wainner RS, Childs JD. Psychometric properties of selected tests in patients with lumbar spinal stenosis. Spine J. 2012;12(10):921-931.

22. Lauridsen HH, Manniche C, Korsholm L, Grunnet-Nilsson N, Hartvigsen J. What is an acceptable outcome of treatment before it begins? Methodological considerations and implications for patients with chronic low back pain. Eur Spine J. 2009;18(12):1858-1866.

23. Fritz JM, Irrgang JJ. A comparison of a modified Oswestry low back pain disability questionnaire and the Quebec back pain disability scale. Phys Ther. 2001;81(2):776-788.

24. Frontera WR, Hughes VA, Lutz KJ, Evans WJ. A cross-sectional study of muscle strength and mass in 45- to 78-yr-old men and women. J Appl Physiol (1985). 1991;71(2):644-650.

25. Macaluso A, De Vito G. Muscle strength, power and adaptations to resistance training in older people. Eur J Appl Physiol. 2004;91(4):450-472.

26. Lindle RS, Metter EJ, Lynch NA, et al. Age and gender comparisons of muscle strength in 654 women and men aged 20-93 yr.J Appl Physiol (1985). 1997;83(5):1581-1587. 
27. Lewandowski AS, Palermo TM, Peterson CC. Age-dependent relationships among pain, depressive symptoms, and functional disability in youth with recurrent headaches. Headache. 2006;46(4):656-662.

28. Walker LS, Greene JW. The functional disability inventory: Measuring a neglected dimension of child health status. J Pediatr Psychol. 1991;16(1):39-58.

29. Behrend C, Prasarn M, Coyne E, Horodyski M, Wright J, Rechtine GR. Smoking cessation related to improved patient-reported pain scores following spinal care.J Bone Joint Surg Am. 2012;94(23):2161-2166.

30. Ashworth J, Green DJ, Dunn KM, Jordan KP. Opioid use among low back pain patients in primary care: Is opioid prescription associated with disability at 6-month follow-up? Pain. 2013;154(7):1038-1044.

31. Ploghaus A, Narain C, Beckmann CF, et al. Exacerbation of pain by anxiety is associated with activity in a hippocampal network. J Neurosci. 2001;21(24):9896-9903.

32. Ruscheweyh R, Albers C, Kreusch A, Sommer J, Marziniak M. The effect of catastrophizing self-statements on pain perception and the nociceptive flexor reflex (RIII reflex). Clin J Pain. 2013;29(8):725-732.

33. Vowles KE, Gross RT. Work-related beliefs about injury and physical capability for work in individuals with chronic pain. Pain. 2003;101(3):291-298.

34. Swinkels-Meewisse IE, Roelofs J, Verbeek AL, Oostendorp RA, Vlaeyen JW. Fearavoidance beliefs, disability, and participation in workers and non-workers with acute low 
back pain. Clin J Pain. 2006;22(1):45-54.

\section{FIGURES}

Figure 1. Flow chart of the medical files analysed.

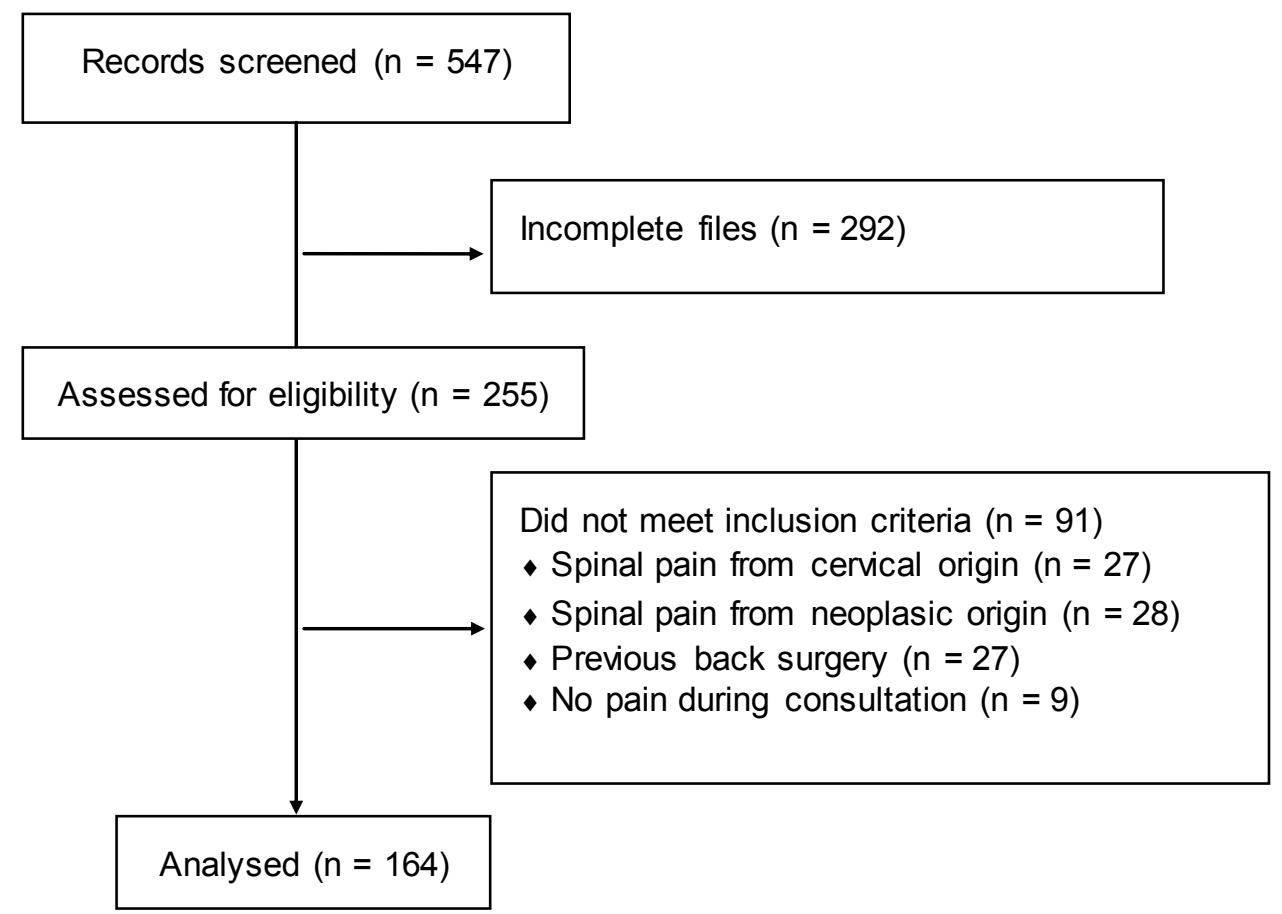


Figure 2. Relationship between pain intensity and physical disability in young and older individuals. Although there was a significant relationship between pain and disability in both young $(\mathrm{r}=0.66, \mathrm{p}<.001)$ and older $(\mathrm{r}=0.44, \mathrm{p}<.001)$ individuals, the relationship between these two variables was significantly higher in young individuals compared to older individuals (Fisher $r$-to-z, $\mathrm{Z}=2.03, \mathrm{p}<0.05$ ). VAS: visual analog scale, ODI: Oswestry Disability Questionnaire.

Young group (22 to 48 years old)

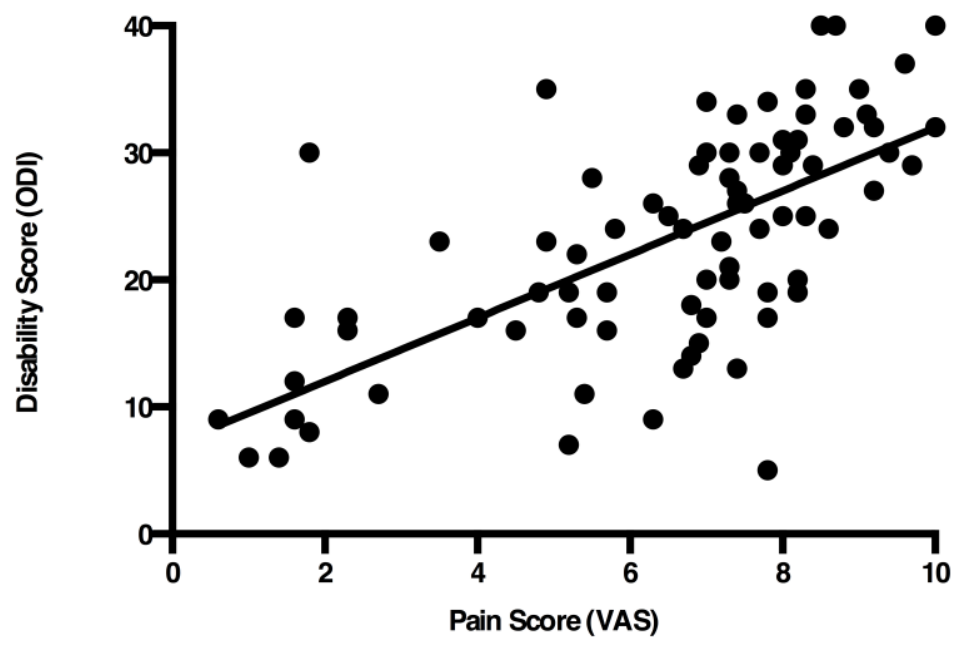

Old group (49 to 90 years old)

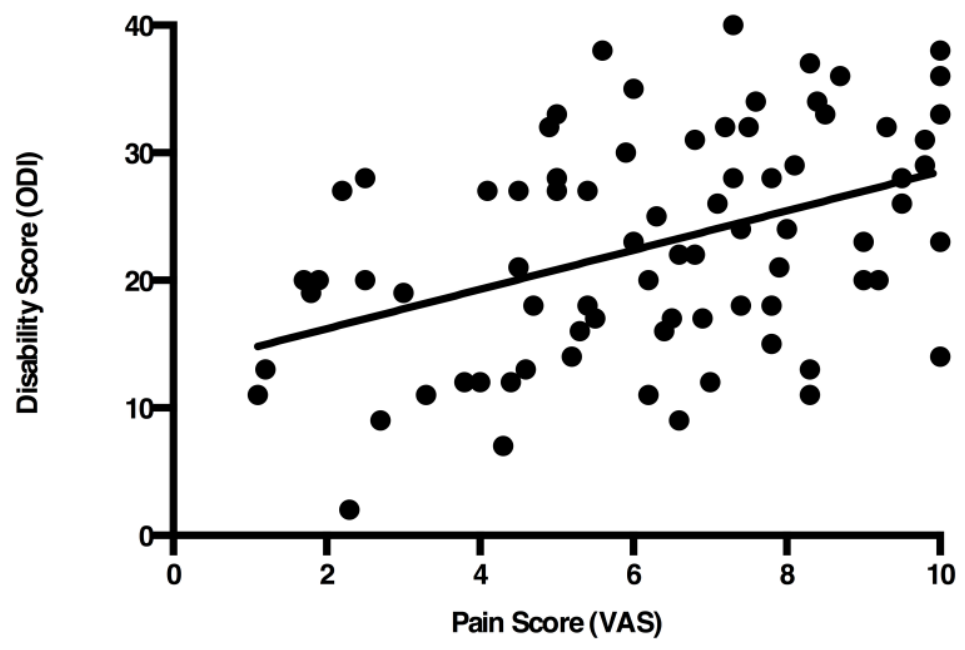


Figure 3. Linear regression equations for young and older individuals. The linear regression model revealed the presence of a significant "Pain X Age group" interaction. VAS: visual analog scale, ODI: Oswestry Disability Questionnaire.

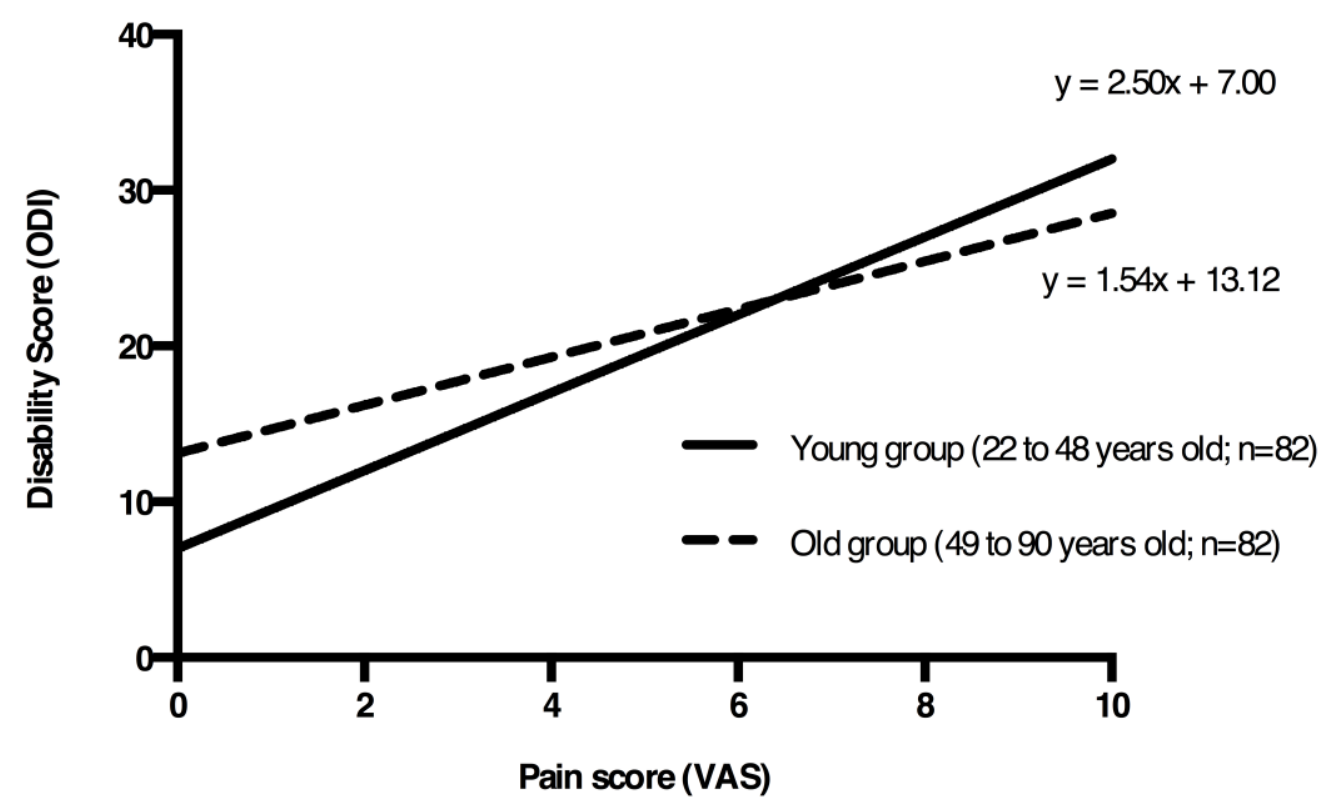

